\title{
LOS RITMOS Y LA RIMA DE LA VERSIFICACIÓN GOETHEANA EN LAS VERSIONES MÉTRICAS DEL FAUSTO EN ESPAÑOL
}

\author{
Stefan Beyer \\ st_beyer@arcor.de \\ Universidad Nacional Autónoma de México
}

\section{Resumen}

Los pocos traductores que han intentado hacer una traducción en verso del Fausto de Goethe no han logrado, en la mayoría de los casos, hacer justicia a la diversidad métrica de la obra del autor alemán. Algunos han abandonado por completo la idea, e.g. Pedro Gálvez en 1984, mientras que otros han creado un poema con métrica tradicional del español tomándose libertades excesivas en cuanto al contenido original del Fausto, tal como sucede en la versión de 1882 de Teodoro Llorente o en las de Valverde (1963) y Silvetti Paz (1970), quienes han empleado, en algunos casos, versos que parecen pentámetros yámbicos sin rima, una medida inusual en la poesía en español y que sin embargo resulta en ocasiones sorprendentemente efectiva. Sin embargo, una traducción que resalta entre todas las versiones métricas existentes del Fausto es la de Augusto Bunge (1926, 1949), quien estudió y analizó cuidadosamente la estructura métrica de esta obra y creó una versión en español que imita las complejas rimas y patrones métricos de Goethe y, a su vez, permanece en gran parte fiel al contenido del texto original en alemán.

\begin{abstract}
"The rhythms and rhyme schemes of Goethe's versification in the metrical versions of Faust in Spanish"

The few translators who have attempted a Spanish verse translation of Goethe's Faust for the most part fail to do justice to the metrical diversity of Goethe's play and therefore they either altogether give up the idea of rendering Goethe's play in poetry, e.g. Pedro Gálvez in 1984, or they create a traditionalist Spanish Faust poem with excessive liberties regarding its content, like, in 1882, Teodoro Llorente. Others have tried
\end{abstract}


to fit Goethe's verse in the Procrustean bed of unrhymed endecasilabos, which has forced them to abridge the original text in numerous passages (Valverde 1963). Moreover, some authors, such as Valverde and Silvetti Paz (1970), translate some passages of Goethe's magnum opus into Spanish verses resembling unrhymed iambic pentameter, a rather unusual approach in Spanish poetry, but nevertheless surprisingly effective. The translation which clearly stands out is the one by Augusto Bunge (1926, 1949), who carefully studied and analyzed the metrical structure of Faust and created a Spanish version which imitates Goethe's complex rhythms and rhyme patterns while remaining largely faithful to the German original.

Palabras clave: Goethe. Fausto. Traducciones en verso. Español.

Keywords: Goethe. Faust. Metrical versions in Spanish. Translation.

Manuscript received on June 30, 2012 and accepted on September 7, 2012. 
Este trabajo es parte de mi interés por emprender la tarea "digna de titanes" (Roviralta 1920:7) de traducir el Fausto de Goethe en verso, para lo cual es preciso antes estudiar a fondo las traducciones existentes, ya que éstas dan pistas esenciales para saber cuáles métodos habría que descartar y cuáles enfoques se deberían adoptar.

En los últimos ciento cincuenta años, se han publicado muchas traducciones del Fausto de Goethe al español, en las cuales se han reflejado, durante largo tiempo, los mismos malentendidos y prejuicios. El personaje de Fausto, por ejemplo, en muchas ocasiones, es representado como un seductor sin escrúpulos, quien, por medio de la magia, sabe procurarse conocimientos y amor. El autor de ese supuesto Don Juan alemán, el "más cuestionable de los clásicos" según José Ortega y Gasset (1932: 46), le parece a los críticos españoles igualmente inhumano que su personaje. Incluso un traductor tan culto como Rafael Cansinos Assens (1957: 1: 252) llega a la conclusión drástica de que Goethe,

todo lo sacrificaba al propio yo: amores, amistades, todo. Era tan buen ecónomo de su dinero que de su adrenalina. Su camino triunfal está sembrado de víctimas incruentas. Su ley es la del más fuerte; quien no es capaz de resistirlo debe sucumbir.

Los malentendidos interculturales en la recepción de Goethe en España en el siglo XIX ya fueron investigados desde hace medio siglo por Udo Rukser (1958) y Robert Pageard (1958).

La historia de las traducciones del Fausto en verso es todavía más asombrosa, porque los secretos de la versificación goetheana no se revelan con facilidad. Además, por lo general, las formas métricas del alemán y del español obedecen a convenciones distintas. En la métrica hispánica, el número de sílabas de un verso constituye el criterio principal, mientras que la distribución y el ritmo de las sílabas acentuadas se emplean para variar el verso. En el siglo de Goethe, la métrica alemana dejó de contar sílabas y se enfocó únicamente en el número de sílabas acentuadas, imitando así en cierta forma las reglas métricas de la antigüedad clásica. En consecuencia, en alemán una serie de versos sí pueden tener números de sílabas variables, aunque en cada verso el número de sílabas acentuadas sea igual. 
No cabe duda de que un Fausto en prosa evoca una idea enteramente distinta de la del original, dado que la métrica de la obra contribuye, de manera vital, a su efecto artístico. En la segunda mitad del siglo XX, la versificación sumamente compleja del Fausto fue investigada en forma meticulosa por Trunz y Ciupke, quienes demostraron hasta qué punto los ritmos goetheanos dan forma a los personajes del Fausto y cómo contribuyen con regularidad a la expresión de sus emociones. Por ejemplo, los frecuentes cambios métricos en los versos del protagonista Fausto indican cómo cambia su estado de ánimo. Además, el uso de una gran variedad de versos irregulares simboliza el individualismo radical de este personaje:

Él solo entre todos los personajes domina todos los tipos de versos [...]. Su discurso se caracteriza sobre todo por el uso de ritmos y de versos irregulares, [que constituyen] los metros de la expresión individual inmediata. (Ciupke 1994: 20)

De manera igual, el Madrigalvers empleado por el diablillo Mefistófeles ilustra la elegancia seductora de este otro personaje. Asimismo, el hecho de que Mefistófeles se adapte a los versos de los demás, y los imite, constituye su estrategia para manipular a los otros personajes (cf. Ciupke 1994: 20).

Goethe escribe en su carta a Schiller del 5 de mayo de 1798 que la versificación del primer Fausto en su forma final le parece un velo mitigador para suavizar la vehemencia de los primeros borradores en prosa de sus tiempos de Sturm und Drang. El poeta weimarano dictó las primeras escenas de su magnum opus, cuando tenía apenas veintitrés o veinticuatro años y estaba buscando formas de expresión literaria más auténticas e inmediatas que las empleadas por muchos de sus contemporáneos, lo cual lo llevó a incorporar en su texto los temas y los géneros populares del siglo XVI. En la segunda fase de la creación del Fausto, la última década del siglo XVIII, es decir, en su fase clasicista, Goethe versificó los últimos pasajes en prosa en el manuscrito original, excepto uno, porque la prosa le parecía insoportable.

Entre los veintiséis metros de la primera parte del Fausto, el más característico es el Knittelvers, ese verso popular e irregular pareado de las farsas de carnaval de Hans Sachs, que Goethe emplea de manera provocadora para producir un efecto casi cómico (cf. Ciupke 1994: 38). En otros pasajes del primer Fausto, se usa el Madrigalvers, versos de cuatro, cinco o seis pies yámbicos con rima pareada, abrazada o alternada. También estrofas como la Stanzenstrophe, una imitación de la octava rima, forman parte de la riqueza métrica del Fausto. Asimismo, el manejo inigualado e irrespetuoso de la rima por Goethe, en casi todos los versos de esta obra de dimensiones épicas, crea una sugestividad y nitidez de la expresión tan grandes que muchos versos 
fáusticos se han vueltos refranes en alemán. Los ritmos del Fausto, sea en el canto melodioso de los arcángeles o en el balbuceo soñador del protagonista cuando expone su credo a su amada ingenua, son tan variados que ni la prosa más artística podría hacerles justicia.

La rima es otro aspecto crucial de la forma inconfundible del Fausto. ¿Cómo traducir versos en los cuales la rima se vuelve objeto del discurso, por ejemplo si afirman en el Walpurgisnachtstraum que la palabra alemana Teufel rima solamente con Zweifel, una rima que además es empleada por el protagonista en su primer monólogo Habe nun, ach..., o la manera poética y bella en la cual sucede el encuentro entre Fausto y Elena (vv. 9367f.), porque la rima es empleada como metáfora del amor, simbolizando un acercamiento entre la modernidad y la antigüedad clásica? En el caso de Goethe, estos efectos poéticos son expresión de su maestría inigualable en el manejo de la rima. No obstante, para el traductor, la rima constituye un obstáculo a veces insuperable; por ejemplo, se puede observar que los traductores, cuando riman, invierten el orden normal de las palabras mucho más frecuentemente que Goethe. Sea como fuere, en el caso del Fausto, el mandamiento de la fidelidad de la traducción no sólo debería entenderse con respecto al argumento de la obra, sino también a su versificación.

El primer autor de una traducción en verso del primer Fausto al español, el poeta valenciano Teodoro Llorente (1882: 31), soñaba con "dar carta de ciudadanía en nuestra patria literaria a la gran creación de Goethe [...]: hay que acomodar la expresión a la índole peculiar de nuestra poética; hay que darle sabor verdaderamente castellano." Llorente probablemente no dominaba muy bien el alemán y la literatura crítica alemana no le interesaba. En las reseñas del siglo XX se hace sentir a veces un tono de mofa ante el "estilo solemne, arcaico y un poco declamatorio" (Pageard 1958: 150) del Fausto de Llorente, que no me parece justificado. Sin embargo, pese a la belleza poética de muchos pasajes, sería más adecuado llamar el Fausto de Llorente no una traducción, sino una obra inspirada por Goethe. Me he ocupado de esta cuestión en forma más detallada en mi estudio sobre las traducciones del Fausto de Goethe (Beyer 2012: 35-38).

Llorente emplea la rima consonante y entre sus versos predominan los octosílabos, decisiones artísticas por las cuales se aleja mucho del texto de origen alemán, tanto respecto a la forma como al contenido. Por ejemplo, un domingo de Pascua, Fausto y su ayudante Wagner dan un paseo, admirando las primeras señas de la primavera. En su larga descripción, Fausto menciona también el río lleno de "barcas alegres" (so manchen lustigen Nachen, Fausto vv. 931-932): 
Wie der Fluß, in Breit' und Länge,

So manchen lustigen Nachen bewegt [...]

La descripción de la naturaleza primaveral y de la gente alegre y pacífica se refleja en los metros empleados por Fausto. Son versos que se parecen a los Knittelverse, con los cuales Fausto se introduce al público al inicio de la "tragedia", solamente sin la típica rima pareada y sin las irregularidades que expresan la alteración del protagonista en aquella primera escena "Noche" (cf. Ciupke 1994: 49). El intento de Llorente (1882: 90) para traducir el discurso de Fausto en octosílabos fracasa, porque esta restricción innecesaria lo obliga a omitir expresiones importantes y además, el ritmo no es el mismo; le falta la tranquilidad pacífica de los primeros días de primavera evocada en el original.

¡Cuántos lleva el ancho río

esquifes empavesados!

Otro ejemplo ilustra todavía más hasta qué punto la traducción de Llorente se vuelve infiel por su métrica y su uso frecuente de la rima consonante. En una de las escenas finales de la primera parte de la obra, Fausto hiere a Valentín en un duelo, con la ayuda de los trucos de magia de Mefistófeles. Cuando Fausto y Mefistófeles han huido, Valentín, muriendo, insulta a su hermana llamándola eine Hur ('una ramera'). Asustada, ella exclama “¡Dios!”, por lo cual el hermano herido la regaña diciéndole que no meta al Señor en el asunto (Fausto v. 3733):

Lass" unsern Herr Gott aus dem Spaß.

Literalmente, Valentín le dice que no meta al Señor en la "broma", pero lo que quiere decir en realidad, de manera irónica, es 'en el asunto', lo cual es obvio en el artículo "spasz" del diccionario de Jakob y Wilhelm Grimm, donde citan el verso 3733 del Fausto y lo explican de la manera siguiente: "im weitesten sinne wie sache, angelegenheit überhaupt, scherzhaft oder ironisch". Además, el verso precedente de Margarita termina en el pronombre demostrativo das; entonces es probable que Goethe utilizara el sustantivo Spa $\beta$, porque las dos palabras riman. La versión de Teodoro Llorente (1882: 292) no es literal ni emplea una paráfrasis, sino que introduce un fraseologismo ("ni arte ni parte") totalmente ajeno al texto de origen:

Dios no tiene arte ni parte

en esto: déjale aparte [...]

El médico argentino Augusto Bunge aprendió de Llorente lo que hay que evitar en su traducción del primer Fausto de Goethe, "tanto en estructuras métricas cuanto en modos de decir" (Bunge 1926: 11). El Fausto de Bunge 
aparece en 1926 en Buenos Aires, después de poco más de un año de trabajo. En 1949 se publica una edición póstuma, bajo los auspicios de Juan C. Probst, la cual contiene varios cambios por parte del traductor, sin duda evidencia de que Bunge siguió trabajando en su obra en las décadas posteriores a la primera edición. En cuanto a la métrica, Bunge tiene un enfoque radicalmente opuesto al de Llorente, y es el primer traductor del Fausto quien verdaderamente se preocupa por una forma métrica adecuada. La meta de Augusto Bunge fue recrear en español lo que él llama las formas "modernistas" de Goethe, es decir, su manejo irrespetuoso de las formas tradicionales prescritas por las poéticas de su tiempo. Al mismo tiempo, quiere lograr una traducción fiel, lo que para Bunge implica "romper con nuestras fórmulas métricas estereotipadas". Bunge está en contra de los versos "uniformes", que constituyen para él un "lecho de Procusto" y prefiere metros irregulares (muy a la manera de Goethe), intentando reproducir "el estilo y el tono correspondientes a cada personaje y a cada situación” (Bunge 1926: 13).

Durante el paseo del Domingo de la Resurrección mencionado arriba, Wagner, el criado de Fausto, admite que él también ha conocido horas de melancolía, pero no entiende el impulso hacia las alturas de Fausto (Fausto vv. 1100-1101):

Ich hatte selbst oft grillenhafte Stunden,

Doch solchen Trieb hab ich noch nie empfunden.

Wagner emplea Madrigalverse con un ritmo muy regular, casi monótono, lo cual expresa su pedantería. En la versión de Bunge (1926: 70), este efecto es recreado por medio de la rima pareada.

También yo tuve algún momento extravagante,

Mas nunca sentí impulso semejante.

Augusto Bunge recrea la irregularidad de los metros y las rimas del Madrigalvers goetheano por medio de una gran variedad de formas métricas, lo que se puede apreciar en la escena del gabinete de estudio, en la cual Mefistófeles aparece por primera vez disfrazado como estudiante andariego. Goethe emplea versos de seis y cinco pies yámbicos (Fausto vv. 1349-1352):

Ich bin ein Teil des Teils, der Anfangs alles war,

Ein Teil der Finsternis, die sich das Licht gebar,

Das stolze Licht, das nun der Mutter Nacht

Den alten Rang, den Raum ihr streitig macht [...]

Bunge (1926: 81) emplea alejandrinos, heptasílabos y endecasílabos con rima variable y en parte imita el ritmo yámbico del Madrigalvers. 
Soy parte de la parte que al principio fue todo,

Parte de las tinieblas que a la luz dieron vida;

La luz dominadora,

La que a la Madre Noche, sin respeto,

El rango y sitio le disputa ahora.

La sensibilidad con la cual Bunge imita los ritmos de Goethe se muestra también en su traducción de un pasaje algo oscuro de la segunda escena del gabinete de estudio (Fausto vv. 1457-1460), que según la erudita interpretación de Hans Arens (1982: 165) se refiere a formaciones de nubes:

Himmlischer Söhne

Geistige Schöne,

Schwankende Beugung

Schwebet vorüber.

El ritmo dactílico de este pasaje se recrea con mucha precisión en el Fausto de Augusto Bunge (1926: 85) — aunque en dos versos largos, en vez de cuatro versos cortos-:

De hijos celestes ideal belleza

Grácil se inclina; leve se esfuma [...]

El pasaje en el cual Fausto explica balbuceando su religión panteísta a su amada ingenua es métricamente uno de los más irregulares de la obra, incluso la rima tan apreciada por Goethe desaparece en esta escena. Ya que sus convicciones divergen de los dogmas de las grandes iglesias, Fausto también emplea un lenguaje muy individualista: se expresa en versos cortos e irregulares, en un tono "hímnico" (cf. Ciupke 1994: 77). Bunge (1926: 191) no sólo imita los ritmos irregulares por medio de la silva, también deja de emplear rima consonante en este pasaje y la reemplaza con rima asonante:

¡No me comprendas mal, mi niña bella!

¿Quién puede osar nombrarle? ¿Quién osara

Decir: yo creo en él? ¿Y quién pudiera

Sentir y osar decirlo: en él no creo?

El que todo lo alienta,

El que todo lo abarca,

¿No está en ti, no está en mí, y todo lo llena?

Allá arriba su cúpula alza el cielo [...]

El Fausto en verso de José María Valverde aparece en 1963. Su enfoque es muy distinto tanto al de Bunge como al de Llorente, ya que Valverde emplea mayoritariamente endecasílabos sin rima (aunque también emplea la silva, por ejemplo en la escena en la cual Fausto expone su credo), de manera que utiliza un estilo de versificación opuesto a la variedad y originalidad goetheanas. Aunque Valverde logra una fidelidad mucho más alta que Llorente, sobre 
todo porque se basa en la erudita traducción en prosa de Rafael Cansinos Assens, pasa por alto los frecuentes cambios métricos del texto de origen, que tanto contribuyen a dar forma a los personajes de la obra. Asimismo, el verso regular se vuelve, en las palabras de Bunge, un "lecho de Procusto", en el cual la idea de los versos goetheanos frecuentemente se mutila. Además, Valverde prescinde del efecto característico que crea la rima en el Fausto de Goethe.

En el "Prólogo en el cielo" (Fausto vv. 243-246), los arcángeles alaban la creación maravillosa en tres estrofas que consisten en ocho tetrámetros cada una, rimados alternadamente con rima aguda y rima llana.

Die Sonne tönt nach alter Weise

In Brudersphären Wettgesang

Und ihre vorgeschriebene Reise

Vollendet sie mit Donnergang.

Se trata de una antigua estrofa que se usaba en el siglo XVII en las iglesias de Alemania para alabar a Dios (cf. Ciupke 1994: 35). La versión de Valverde, en endecasílabos sin rima, es sumamente fiel, ya que emplea más sílabas que Goethe, pero no evoca para nada el tono ceremonial y grandioso de la canción religiosa:

Con la antigua armonía el sol resuena

entre el canto fraterno y a porfía

de las esferas; $y$ anda, como un trueno,

hasta el final de la prescrita senda.

Por otra parte, en el mismo pasaje en la traducción de Bunge (1949: 11), unos pareados endecasílabos con rima consonante imitan de manera muy fiel la forma de las estrofas goetheanas.

El sol canta la vieja melodía

Entre esferas hermanas a porfía.

Y cierra el viaje que le fue prescrito

Como trueno a través del infinito.

El argumento aparece de forma ligeramente alterado aquí, pero sí se reproduce la idea del texto de origen.

La necesidad de omitir palabras al emplear casi únicamente el verso endecasílabo resalta claramente si comparamos el Fausto de José María Valverde con la versión en prosa de su predecesor Rafael Cansinos Assens. En su primer encuentro con Wagner, Fausto se sirve de la metáfora der Menschheit Schnitzel kräuseln para ilustrar la retórica vacía de su criado. Schnitzel aquí no se refiere al famoso platillo vienés, sino a pedacitos de papel que se rizan, de manera decorativa, con tijeras. Gerhard Sauder, en la edición de Múnich de 1987 (tomo 1.2: 753) observa que la metáfora de las rizas de papel viene 
de una técnica utilizada en la construcción de pelucas barrocas. Fausto aquí se sirve de una imagen literaria sumamente atrevida y apenas comprensible para ilustrar qué tan aburrido le parece el lenguaje figurativo que se aprende de la escuela clásica. Cansinos traduce el sustantivo Schnitzel por "virutas", término que, por lo regular, se refiere a la madera, no al papel. En su versión (Cansinos 1957: 1: 365), las virutas además se rizan: "en las que rizáis virutas a la Humanidad". 'Rizar' sí corresponde a kräuseln y técnicamente parece adecuado también en el caso de la madera, pero se aleja demasiado de la imagen que crea Fausto en el texto de origen. No obstante, Cansinos explica la "metáfora oscura" en una larga nota pertinente. José María Valverde (1963: 20) imita la solución de Cansinos sin la nota al pie, pero condensa la idea casi hasta la incomprensibilidad para cumplir con su endecasílabo:

En que a la Humanidad sacas virutas [...]

José María Valverde emplea el verso endecasílabo incluso en pasajes donde Goethe emplea versos mucho menos largos, por ejemplo en el canto del Espíritu de la tierra. En la escena "Noche" (Nacht), el Erdgeist revela a Fausto que su actividad consiste en originar el ciclo de la vida y toda acción en la tierra. El espíritu emplea versos trocaicos, alternados con versos yámbicos, lo que crea un ritmo muy agitado, que evoca el mismo movimiento del espíritu, primero con rima cruzada y al final con rima pareada (Fausto vv. 501-503).

In Lebensfluten, im Tatensturm

Wall' ich auf und ab,

Wehe hin und her!

Valverde (1963: 19) no recrea el juego rítmico del Erdgeist, y en consecuencia no se da la idea del movimiento del espíritu. Además, el encabalgamiento nivela el ritmo del pasaje en alemán todavía más.

En rebose de vida, en tempestad

de acción, yo subo y bajo en oleadas,

y me agito de un lado para otro.

Sin embargo, Valverde con regularidad recrea los ritmos yámbicos de varios versos goetheanos por medio de endecasílabos heroicos (cf. Navarro 1959: 51), cuyos pies trocaicos regulares evocan un Blankvers alemán. Por ejemplo, en la famosa escena con el alumno, Mefistófeles observa irónicamente que, en la metafísica, hay que saber captar, de manera profunda, lo que "no cabe en el cerebro humano" (Fausto, vv. 1951-1952):

Da seht daß ihr tiefsinnig faßt,

Was in des Menschen Hirn nicht paßt. 
José María Valverde (1963: 56) traduce en versos endecasílabos heroicos, los cuales evocan el mismo ritmo yámbico regular:

Veréis cómo captáis con honda mente

lo que en cabeza humana nunca cabe.

Este tipo de versos ocurre tan frecuentemente en la versión de Valverde que debe ser un efecto intencional. De igual manera, Norberto Silvetti Paz usa estos ritmos "yámbicos", e incluso me parece que lo anuncia en su introducción, cuando dice que empleó "el verso blanco" (Silvetti Paz 1970: 24). Considero que no sólo se refiere a versos sin rima aquí, sino que en realidad habla del Blankvers, es decir, versos de cinco pies métricos yámbicos. En la "Noche de Walpurgis" encontré un ejemplo de esto (Fausto, vv. 4115) cuando al ver la multitud de brujas que suben al Brocken, Fausto exclama:

Heiß' ich mir das doch eine Messe!

El ritmo en la versión de Silvetti Paz (1970: 357) aquí se asemeja al de Goethe:

Jamás he visto feria semejante!

En la misma escena, algunos momentos antes (Fausto, vv. 4042), Mefistófeles ha desviado a Fausto de la corriente principal de las brujas y los brujos dirigiéndose al aquelarre, para llevarlo a una zona más tranquila de la fiesta satánica. Esta decisión de Goethe, de no incluir la escena del aquelarre en su Fausto, ha sido tema de controversias muy prolongadas en la filología alemana. Para velarlo, Mefistófeles pretende que le gustaría un poco de tranquilidad:

Wir wollen hier im Stillen hausen.

En la versión de Silvetti Paz (1970: 350), Mefistófeles expone a Fausto las ventajas de caminar tranquilamente de una fogata a otra, en vez de asistir a la veneración del diablo, con el siguiente endecasílabo heroico:

$\mathrm{Y}$ aquí nos quedaremos en sosiego.

El Fausto de Pedro Gálvez (1984) no se basa realmente en principios métricos, ya que los versos muchas veces no tienen ritmo y su longitud varía mucho, como se puede ver en su versión bastante abstracta (Gálvez 1984: 88) de la observación de Mefistófeles acerca de la metafísica que citamos arriba:

Procurando entender perspicazmente

aquello que no se aviene con el cerebro humano [...]

También en la discusión entre Fausto y Mefistófeles después del primer encuentro entre Fausto y Margarita, los versos parecen extremadamente irregulares y carecen de ritmo. Mefistófeles quiere convencer a Fausto de que 
no conviene conquistar a Margarita apresuradamente, sino que Fausto y él deberían "acomodarse" al ardid (Fausto vv. 2654-2658):

Jetzt ohne Schimpf und ohne Spaß.

Ich sag' euch, mit dem schönen Kind

Geht's ein- für allemal nicht geschwind.

Mit Sturm ist da nichts einzunehmen;

Wir müssen uns zur List bequemen.

Se trata de versos de cuatro pies, en su mayoría yámbicos, con rima pareada. En la versión de Pedro Gálvez de este pasaje con métrica bastante regular en el original alemán (Gálvez 1984: 133), tanto los ritmos como la medida de los versos varían mucho en el español:

$\mathrm{Y}$ os digo ahora, sin insulto ni chirigota:

con esa niña hermosa el apresuramiento nada os sirve,

nada conquistaréis con un asalto;

habremos de recurrir a argucias y artimañas.

También en la discusión siguiente entre el catedrático hechizado y su acompañante, la versificación de Goethe no se refleja en los versos de Gálvez. Mefistófeles se burla de la locura amorosa de Fausto, comparándola con fuegos artificiales (Fausto, vv. 2862-2864):

So ein verliebter Tor verpufft

Euch Sonne, Mond und alle Sterne

Zum Zeitvertreib dem Liebchen in die Luft.

Nuevamente se trata de Madrigalverse muy regulares, lo cual no se refleja en la traducción de Gálvez (1984:144):

Un loco prendado de tal suerte os hará estallar por los aires

sol y luna y todas las estrellas,

para solaz y esparcimiento de su amada.

En su traducción, Gálvez emplea un sistema de versificación, cuyo único principio me parece ser la distribución exacta de las ideas en los versos. En realidad, en muchos aspectos, se trata de una traducción en prosa impresa en forma de versos, por lo tanto es una traducción línea por línea con pocos criterios verdaderamente métricos.

Helena Cortés (2010) emplea versos muy largos y ocasionalmente rima asonante, un enfoque que le permite traducir muchos pasajes de manera fiel. La rima asonante que usa en el "Sueño de la noche de Walpurgis" (Walpurgisnachtstraum) imita, en cierta manera, la regularidad excesiva de la versificación de la escena en el texto de origen, como muestra la estrofa del bailarín (Fausto vv. 4331-4334). Según los comentaristas más destacados, se refiere a los filósofos, que son comparados a Rohrdommeln (botaurus stellaris), el "avetoro común", 
un tipo de garza que produce gritos graves, que suenan como los bramidos de un toro, y que hace ruidos parecidos a un tambor hindú (cf. Beyer 2012: 296).

Da kommt ja wohl ein neues Chor?

Ich höre ferne Trommeln.

Nur ungestört! Es sind im Rohr

Die unisonen Dommeln.

La mayoría de los traductores traduce los Rohrdommeln como 'alcaravanes', en España un ave más conocida. Cortés (2010: 299) traduce el "Sueño de la noche de Walpurgis" en estrofas de octosílabos cruzados, lo que me parece una buena solución.

¿Se oye en los juncos un coro?

Siento lejanos tantanes.

Ni caso: graznan tediosos

los vanos alcaravanes.

Otro ejemplo de esta estrategia adaptada por Cortés (2010: 43) es su versión del canto de los arcángeles. Los tetrámetros goetheanos son traducidos por medio de dodecasílabos regulares, versos suficientemente largos para lograr una alta fidelidad:

El sol entona, siguiendo antiguo uso, su canto con las esferas en concurso, y su viaje, ya prescrito de antemano, conduce a su culmen con tonante paso.

Además, la rima asonante recrea, de manera sutil, la rima de Goethe y al mismo tiempo no impide ni la expresión natural, ni la traducción fiel.

En resumen, los autores de traducciones métricas del Fausto a lo largo de los últimos ciento cincuenta años han usado formas de versificación muy distintas, sin embargo, nadie ha hecho justicia a la gran diversidad métrica del texto de origen. Hasta la fecha, la única traducción que se acerca a la riqueza de los metros y ritmos goetheanos es la de Augusto Bunge, la cual curiosamente sólo fue publicada por una editorial académica, de muy corto tiraje, a mediados del siglo XX. La gran ventaja del enfoque de Bunge es que él aprovecha la irregularidad de las formas métricas de Goethe, ya que ese manejo irrespetuoso de las formas tradicionales por el poeta de Weimar en el texto alemán permite usar un lenguaje y un estilo más naturales del que ofrecen las formas fijas. Valdría la pena hacer una reedición de la versión de Bunge o una nueva versión siguiendo sus pasos. En cuanto a la rima, la rima asonante constituye un recurso todavía poco explotado en las traducciones métricas de Goethe al español que tal vez un día permita lograr tanto una gran fidelidad en cuanto al contenido, como dar una idea más cercana del efecto estético del original. 


\section{Bibliografía}

BAEHR, Rudolf. (1989) Manual de versificación española. Traducción de K. Wagner, y F. López Estrada. Madrid: Gredos.

BEYER, Stefan. (2012) El Fausto de Goethe en español. Estudio crítico de sus traducciones. Tesis de doctorado. Universidad Nacional Autónoma de México.

BungE, Augusto (traductor) \& Johann Wolfgang von Goethe. (1926) Fausto: Tragedia. Primera parte. Buenos Aires: L. J. Rosso y Cía.

Bunge, Augusto (traductor) \& Johann Wolfgang von Goethe. (1949) Fausto. 2a ed. Sección Anglogermánica del Instituto de literatura de la facultad de filosofía y letras de la Universidad de Buenos Aires.

CANSINOS ASSENS, Rafael (traductor) \& Johann Wolfgang von Goethe. (1957) Obras completas. 3 tomos. Edición facsimilar realizada a partir de la edición de 1957. Madrid: Aguilar 2003.

CIUPKE, Markus. (1994) Des Geklimpers vielverworrner Töne Rausch: Die metrische Gestaltung in Goethes "Faust". Göttingen: Wallstein Verlag.

CORTÉs GABAUDAN, Helena (traductora) \& Johann Wolfgang von Goethe. (2010) Fausto. Edición bilingüe. Madrid: Abada.

Das deutsche Wörterbuch von Jacob und Wilhelm Grimm im Internet. Versión electrónica: <www.woerterbuchnetz.de/DWB>

GAIER, Ulrich. (2002) Kommentar zu Goethes Faust. Stuttgart: Reclam.

GÁLVEZ, Pedro (traductor) \& Johann Wolfgang von Goethe. (1984) Fausto. Introducción de José Miguel Mínguez Sender. Barcelona: Bruguera.

GOETHE, Johann Wolfgang von. (c. 1775) Urfaust. En: Sämtliche Werke (Münchner Ausgabe). Tomo 1.2. Gerhard Sauder (ed). 1987. Múnich: Hanser. Btb 2006.

GoETHE, Johann Wolfgang von. (1808) Faust. 2 vol. Albrecht Schöne (ed.) Frankfurt am Main: Deutscher Klassikerverlag, 2005.

LLORENTE, Teodoro (traductor) \& Johann Wolfgang von Goethe. (1882) Fausto: Tragedia de Juan Wolfgango Goethe. Primera parte. Barcelona: Montaner y Simon. Reproducción facsimilar de la edición de 1905. México D.F.: Editorial Coyoacán, 2005.

Navarro Tomás, Tomás. (1959) Arte del verso. México: Colección Málaga, 1977. ORTEGA y GASSET, José. (1932) Carta a un alemán: pidiendo un Goethe desde dentro. Brief an einen Deutschen: Um einen Goethe von innen bittend. Madrid: Biblioteca nueva. Fundación Goethe. Fundación Ortega y Gasset, 2004.

PAgEARD, Robert. (1958) Goethe en España. Traducción de Francisco de A. Caballero. Madrid: Instituto Miguel de Cervantes de Filología Hispánica.

REYES, Alfonso. (1932) "Carta a Eduardo Mallea sobre el Goethe de Ortega y Gasset". En: Obras Completas de Alfonso Reyes. Tomo XXVI. México: Fondo de Cultura Económica, 2003. Pp. 439-445. 
RovirAlTA, José (traductor) \& Johann Wolfgang von Goethe. (1920) Fausto. Barcelona: Ibérica.

RovirAlTA, José (traductor) \& Johann Wolfgang von Goethe. (1920) Fausto. Manuel José González y Miguel Angel Vega (eds). Decimotercera ed. Madrid: Cátedra, 2007.

RUKSER, Udo. (1958) Goethe in der hispanischen Welt. Stuttgart: J.B. Metzlersche Verlagsbuchhandlung. Citado por la traducción de Carlos Gerhardt: Goethe en el mundo hispánico. México: Fondo de Cultura económica, 1977.

SilvetTi PAZ, Norberto (traductor) \& Johann Wolfgang von Goethe. (1970) Fausto. Buenos Aires: Editorial Sudamérica.

VALVERDE, José María (traductor) \& Johann Wolfgang von Goethe. (1963) Fausto. Introducción de Francisca Palau Ribes. $2^{a}$ ed. Barcelona: Planeta 2005.

\section{NOTA BIOGRÁFICA / BIONOTE}

Stefan Beyer estudió letras inglesas, letras francesas y enseñanza de idiomas en la Technische Universität Berlin y la Université Stendhal, Grenoble; fue docente a nivel licenciatura en la Facultad de Letras de la Technische Universität Berlin y acaba de terminar sus estudios de doctorado en la Universidad Nacional Autónoma de México. Sus intereses principales en investigación han sido la crítica de la traducción y la historia de la traducción, la intertextualidad y el drama en la educación.

Stefan Beyer studied English and French literatures, linguistics and language teaching (Technische Universität Berlin and Université Stendhal (Grenoble). He is a former lecturer in English literature (Technische Universität Berlin). He recently completed a doctorate (Universidad Nacional Autónoma de México). His scholarly interests include translation criticism and translation history, intertextuality (English Neoclassicism) and drama in education. 